\title{
Particle Physics at PSI
}

\author{
A. Signer ${ }^{1,2 \dagger}$, K. $\operatorname{Kirch}^{1,3 \star}$ and C. M. Hoffman ${ }^{\star}$
}

1 Paul Scherrer Institute, Villigen PSI, Switzerland

2 University of Zurich, Physik-Institut, Zurich, Switzerland

3 ETH Zurich, Institute for Particle Physics and Astrophysics, Zurich, Switzerland

$\dagger$ adrian.signer@psi.ch, $¥$ klaus.kirch@psi.ch

$\star$ Retired from University of New Mexico, Albuquerque, USA

and Los Alamos National Laboratory, USA

PAUL SCHERRER INSTITUT

(2)

Review of Particle Physics at PSI

doi:10.21468/SciPostPhysProc.5

\begin{abstract}
Particle physics results of constant value and significant impact have been obtained at PSI, and several efforts are presently ongoing and expected to deliver new findings in the near future. In this special SciPost volume we collect them together in a concise manner. Not yet included are ideas for future facility upgrades or completely new experimental efforts, but we are set to extend this volume in the years to come.
\end{abstract}

(c) (1) Copyright A. Signer et al.

This work is licensed under the Creative Commons

Attribution 4.0 International License.

Published by the SciPost Foundation.
Published 06-09-2021

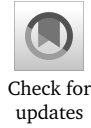

doi:10.21468/SciPostPhysProc.5.001

The intensity frontier of particle physics uses intense particle beams to perform precision tests of the Standard Model (SM) and to search for rare processes forbidden in the SM. This approach is complementary to the energy frontier. The proton accelerator facility at the Paul Scherrer Institute (PSI), with its ring cyclotron [3] generates high-intensity beams of pions, muons and ultracold neutrons. In this special SciPost volume, a collection of articles describes past, present and future experiments using these beams at the intensity frontier, as well as a description of the facility. Ideas for future facility upgrades or completely new experimental efforts are not included here, but we are ready to extend this volume in the years to come.

PSI is the largest federal research institute in Switzerland. It belongs to the ETH domain and serves as a national laboratory to provide large-scale research infrastructure, such as accelerators, that are beyond the means of a single university. PSI was formed in 1988 by the merger of two predecessor institutions: EIR (Eidgenössisches Institut fur Reaktorforschung) and SIN (Schweizer Institut für Nuklearforschung). SIN was founded to host Switzerland's "pion factory", a high-intensity proton accelerator complex with target stations and secondary beam lines. We use the term "PSI" to include facilities and activities at both SIN and PSI.

The proton accelerator facility at PSI produced its first pions shortly after it went into operation in 1974. The design of the high-intensity ring cyclotron was novel and innovative, aiming at a proton current of $100 \mu \mathrm{A}$ at a kinetic energy of $590 \mathrm{MeV}$. The accelerator has been continuously improved and upgraded and has far outperformed these design goals. It is currently 
among the world's most powerful proton accelerators, producing an average beam power of up to 1.4 MW, with a beam current of over $2 \mathrm{~mA}$. Several low-momentum beams of pions and muons are produced with the highest intensities available [4]. In the last 10 years, a dedicated neutron spallation target has been added, which serves as a high-intensity source of ultracold neutrons [5]. As new experiments need even higher intensities to reach their ambitious goals, plans to further upgrade the facility are under discussion.

Many of the particle physics experiments performed at PSI use the high beam fluxes to precisely measure the parameters of the SM, or to search for the possible existence of physics beyond the SM with improved sensitivity. While the results from these studies have been published in many articles and journals over the years, there has been no review to date collecting all of these in one volume. This SciPost Volume aims to fill that void.

Peter Truöl ${ }^{1}$ published a monograph in 2007 [2] entitled Myonen und Pionen in Teilchenphysik und Anwendungen - 30 Jahre experimentelle Forschung an der Schweizer Mesonenfabrik in Villigen. This was, unfortunately, only available in German, and was meant for a different audience than this volume. Peter Truöl, together with one of the editors of this volume (KK), wanted to update and extend this nice booklet into a major review article aimed at an international audience of interested particle physicists. While that project was never realized, the present editors teamed up to assemble this special volume. We distributed much of the work to the many colleagues who are actually much better able to write individual parts of this review, reporting on their own work. We are grateful to all of the authors who accepted our request, and contributed their articles.

When we started this project some time ago, we asked two very dear colleagues, Claude Petijean and Manfred Daum, to serve as guinea pigs. They wrote several example contributions, which were discussed and used to help fix the instructions and templates for the other authors. Claude and Manfred also wrote several final contributions and served as critical reviewers for many other papers. In addition, many other authors and colleagues served as anonymous referees for the contributions to this special volume. We give a huge "thank you" to all those who helped!

We hope that this volume will remain as a reference for the achievements in particle physics at PSI, and expect that many future contributions will extend this collection.

Klaus Kirch, Adrian Signer, Cy Hoffman

\section{References}

[1] P. A. Zyla et al., Review of particle physics, Progr. Theor. Exp. Phys. $083 C 01$ (2020), doi:10.1093/ptep/ptaa104.

[2] P. Truöl, Myonen und Pionen in Teilchenphysik und Anwendungen - 30 Jahre experimentelle Forschung an der Schweizer Mesonenfabrik in Villigen, Naturforschende Gesellschaft in Zürich (2007), ISSN 0379-1327.

[3] J. Grillenberger, C. Baumgarten and M. Seidel, The high intensity proton accelerator facility, SciPost Phys. Proc. 5, 002 (2021), doi:10.21468/SciPostPhysProc.5.002.

[4] D. Kiselev, P. A. Duperrex, S. Jollet, S. Joray, D. Laube, D. Reggiani, R. Sobbia and V. Talanov, The Meson Production Targets in the high energy beamline of HIPA at PSI, SciPost Phys. Proc. 5, 003 (2021), doi:10.21468/SciPostPhysProc.5.003.

\footnotetext{
${ }^{1}$ Peter Truöl (1939-2020) was professor of experimental particle physics at the University of Zurich and chairman of the particle physics advisory committee at PSI for ten years, 1993-2002, after which one of the volume editors (CMH) took over this duty for another long period, 2002-2014.
} 
[5] B. Lauss and B. Blau, UCN, the ultracold neutron source - neutrons for particle physics, SciPost Phys. Proc. 5, 004 (2021), doi:10.21468/SciPostPhysProc.5.004. 